EOMmUn: Communication et organisation

Or

La communication, dimension oubliée de l'intelligence économique

\title{
Entrevue avec Dominique Wolton
}

Nicolas Moinet

\section{OpenEdition}

Journals

Édition électronique

URL : http://journals.openedition.org/communicationorganisation/3918

DOI : 10.4000/communicationorganisation.3918

ISSN : $1775-3546$

Éditeur

Presses universitaires de Bordeaux

Édition imprimée

Date de publication : 1 décembre 2012

Pagination : 149-152

ISBN : 978-2-86781-772-4

ISSN : 1168-5549

Référence électronique

Nicolas Moinet, « Entrevue avec Dominique Wolton », Communication et organisation [En ligne],

42 | 2012, mis en ligne le 01 décembre 2013, consulté le 20 avril 2019. URL : http://

journals.openedition.org/communicationorganisation/3918; DOI : 10.4000/

communicationorganisation.3918 


\section{Deux entretiens réalisés par Nicolas Moinet}

\section{Entrevue avec Dominique Wolton}

Dominique Wolton est Directeur de Recherche de Classe Exceptionnelle au CNRS où il dirige l'Institut des Sciences de la Communication (ISCC) qu'il a créé en juin 2007. L'ISCC est une structure transverse unique et pluridisciplinaire organisée autour de cinq grands domaines : langage et communication ; communication politique, espace public et société ; mondialisation et diversité culturelle; information scientifique et technique ; sciences, techniques et société. Dominique Wolton est l'auteur de nombreux ouvrages sur la communication. Parmi eux, citons notamment : L'autre mondialisation (Flammarion, 2004), Il faut sauver la communication (Flammarion, 2005), Informer n'est pas communiquer (CNRS Editions, 2009) et Indiscipliné-La communication, les hommes et la politique(Odile Jacob, 2012).

Née au début des années 90, l'intelligence économique s'est développée avec la société de l'information mais depuis plusieurs années, tout indique que ce paradigme n'est plus de mise. La société de la connaissance est mise en avant et la communication apparaît être la clé de voûte de l'intelligence économique. Comment analysez-vous plus globalement cette évolution ?

L'information est la vraie victoire du $\mathrm{XX}^{\mathrm{e}}$ siècle. Tant et si bien qu'on a pu parler de révolution de l'information ou même de société de l'information. Mais cette dynamique butte sur deux obstacles : la communication et les connaissances. Avec la généralisation de l'information, la diversité des récepteurs, leur sens critique et la mondialisation, tout se complique. On avait, il est vrai, une vision simple de l'information réduite à un message, le plus souvent univoque, et celle d'un récepteur finalement peu complexe. Avec une sorte de continuum entre les deux et l'idée que l'information plus abondante, et rapide, devait créer plus de communication. C'était le modèle universel de la communication. Il réclame un aggiornamento. Quant au second obstacle sur lequel butte la révolution de l'information, force est de constater qu'il n'est pas plus simple, c'est celui des connaissances. D'où ce décalage croissant qui est ressenti entre le sentiment d'être un géant en 
matière d'informations et un nain en matière d'action. Car pour conduire à l'action, l'information doit devenir connaissance.

\section{Dès lors, que se passe-t-il dans les organisations et comment peuvent-elles relever le défi de la communication et du management des connaissances ?}

À côté de l'idéal de la « communication-partage », recherché par tous, de la réalité dominante de la "communication-transmission ", où la hiérarchie communique de haut en bas, se développe la « communication-négociation », où l'émetteur doit toujours tenir compte de l'intelligence des récepteurs et accepter un retour critique. Car la crise rend les salariés sceptiques et la réalité s'impose, rendant caduques les formules aseptisées, et les directions s'en trouvent plus ou moins désacralisées. Tout est trop instable, incertain, et va trop vite, dans un environnement le plus souvent hostile. Les salariés, quel que soit leur niveau hiérarchique, sont également beaucoup plus informés. À l'extérieur, avec la multitude de sources d'informations et d'interactions, ils savent tout (ou presque) sur la réalité économique, financière et commerciale, et n'oublient rien en franchissant les murs de l'entreprise, ce qui représente une rupture radicale. Le rapport intérieur/ extérieur a changé, et c'est souvent la culture de l'extérieur qui s'impose. Il y a donc un décalage entre la vitesse et la surinformation du citoyen, et l'incertitude qui règne dans l'organisation. En outre, l'organisation étant une communauté d'hommes, elle doit en plus gérer toutes les relations humaines avec leurs forces, faiblesses et turpitudes qui n'ont rien à voir avec l'information et la communication. La problématique universelle du pouvoir n'est pas soluble dans la communication. La « transparence du fonctionnement » de l'organisation et son organigramme n'ont rien à voir, souvent, avec la réalité du fonctionnement des rapports sociaux...

Par ailleurs, contrairement aux apparences, la généralisation des sites, blogs, tweets, et toutes les formes contemporaines de communication ne facilitent pas toujours la communication interne. Ces échanges en réseau et l'expression facilitée par ces technologies ne sont pas forcément compatibles avec les jeux de pouvoir, économiques, financiers, techniques ou commerciaux. L'entreprise ne peut pas être un espace égalitaire et, d'ailleurs, de l'espionnage à la traçabilité, tout se termine parfois devant les tribunaux. Ainsi, l'omniprésence de l'information sur tous les supports, les révélations, accompagnées de rumeurs le plus souvent alarmistes, laissent une marge de manœuvre très faible aux dirigeants et expliquent la tendance à renforcer le secret, parfaitement contradictoire avec l'idéologie de la transparence et de la participation qui domine par ailleurs dans le discours sur le « management moderne». 


\section{Ne sommes-nous pas alors engagés dans un cercle vicieux voire une impasse?}

Non, car face à ces ruptures, il existe une marge de manœuvre. Réaliser que tous les salariés sont critiques, sceptiques et surinformés doit faire glisser le management du côté de la vérité et non plus de la langue de bois. Essayer la confiance demande concrètement de valoriser les directions de la communication tout en remettant à leur place les directions financières, utiliser l'intelligence créatrice de tous qui ne demande qu'à se manifester, partager des informations, écouter d'autres logiques et propositions... bref, apprendre à négocier, c'est-à-dire à communiquer autrement. Et admettre que l'organisation, comme la société, est un lieu de cohabitation. Faire confiance aux uns et aux autres pour aller vers un modèle de communication moins vertical, plus horizontal et authentiquement interactif. Mais attention : mieux mobiliser l'intelligence de tous ne veut pas dire qu'il faille transformer les organisations en phalanstères. Chacun doit rester à sa place avec trois logiques à faire cohabiter : la hiérarchie, l'égalité et la négociation. Autrement dit, l'omniprésence de l'information oblige à valoriser la fonction de communication comme négociatrice entre des points de vue contradictoires. Maintenir l'hétérogénéité dans l'entreprise, comme dans la société, est indispensable pour éviter le risque d'anomie. C'est le triomphe même de l'information qui crée ce changement de modèle de communication dans l'entreprise et ailleurs. Cela ne suffit pas à changer la réalité, mais permet de mieux reconnaître l'apport des uns et des autres, la complexité de la réalité et la cohabitation de niveaux d'expériences.

Dans ce contexte de ruptures et de défis, comment se positionne justement le monde académique ? Si vous appelez à plus d'indiscipline, est-ce à dire qu'il aurait failli dans sa mission d'empêcheur de penser en rond ?

Le monde académique, de la recherche et de la connaissance quoiqu'on dise est écrasé depuis 30 ans par le monde de l'information, des médias, de l'événement. L'information n'a pas tué la connaissance, elle l'a marginalisée. Et le milieu académique porte une responsabilité dans cette marginalisation. C'est l'information, par sa victoire, qui a englouti le culturel et la connaissance avec la vitesse, la brièveté, la concurrence... Et pourtant, plus il y a d'informations, plus on a besoin de connaissances pour les expliquer et les contextualiser.

En d'autres termes, les universitaires ne peuvent plus refuser la révolution de l'information et se réfugier dans une tour d'ivoire aristocratique. En même temps, ils doivent défendre le rôle essentiel de la culture, des connaissances, qui ne doit surtout pas se confondre avec la facilité d'accès aux banques de données. La connaissance échappe, par nature, au monde de l'information interactive. La lenteur contre la vitesse. Le problème n'est pas le volume d'informations, mais les connaissances pour les traiter. D'ailleurs, les 
universitaires sont des intermédiaires indispensables, comme les journalistes doivent le rester pour l'information, dont le monde de l'information ne peut plus se passer. À quand le retour de l'érudition, de la culture, de l'étendue des savoirs transverses pour compenser cette illusion d'une " connaissance évènementielle » sans profondeur dominée par la tyrannie de l'événement ? Internet, compte tenu du volume d'informations accessibles, n'a en général pas plus de dix années de profondeur. Mais attention : les informations et les connaissances ne suffisent pas. Il faut en plus prendre en compte les cadres culturels et idéologiques. Car au-delà de ce qui est gentiment appelé " la communauté internationale de la science et des connaissances ", les rapports de force, la concurrence et les différentes visions du monde seront de plus en plus visibles, avec la nécessité de passer d'un monde unique de la science et des connaissances, si tant est qu'il ait jamais existé, à un monde pluriel dont il faudra organiser la cohabitation. En fait, la généralisation de l'information, son abondance, la vitesse de traitement et l'accessibilité généralisée sont à la fois un facteur d'émancipation et un facteur de complexification fondamental du fonctionnement de nos sociétés. Les systèmes d'information, et les réseaux, sont rationnels, les Hommes, les sociétés et les cultures, jamais... 